\title{
ON THE SHORT PERIOD TERMS IN THE UT1 AND THOSE IN THE POLAR MOTION
}

\author{
SHIGETAKA IIJIMA and SÊICHI OKAZAKI \\ Tokyo Astronomical Observatory, Japan
}

\begin{abstract}
Short period terms ranging from 0.3 to $6.0 \mathrm{yr}$. are resolved from $13.5 \mathrm{yr}$ data of UT1 and polar motion on periodograms, considering the mutual interference effects caused by truncation of data. Beside principal terms known well, 26 month term is reconfirmed but any Chandlerian term is not found in UT1. Minor amplitude terms are found around $0.48,0.86,1.36,1.58,3$, and $4 \mathrm{yr}$ in UT1, and $1.12,1.26$, and $1.43 \mathrm{yr}$ in polar motion.
\end{abstract}

The spectrum of periodic terms for amplitude against period is obtainable by means of the method of least squares, and this method is proved identical with the using Fourier series calculation when the total lapse of time covered by the data is sufficiently long compared with the period of the periodic terms in question. According to the spectrum calculation in this way, a series of data represented by a pure line spectrum, for instance, is calculated as a spectral form consisting of the central peak of a certain width accompanied by a series of nearby peaks with smaller amplitude on both sides. The spectral form can be represented by $|\sin u / u|$ around $u=0$, when $u$ is written for $(N \tau) /(p \tau) \cdot \pi \cdot \Delta p / p$, where $N \tau$ and $p \tau$ are the total lapse of time and the period of the central peak respectively, $\tau$ being the datum interval. This means that the spectrum calculation for a series of data composed of several periodic terms adjacent to one another leads to an erroneous result from mutual interference in the calculation.

Taking these relations into consideration, short period terms ranging from 0.3 to $6.0 \mathrm{yr}$ in the UT1 and those in the polar motion are resolved from data of (UT2-A3) and those of $x$ and $y$ respectively, all covering $13.5 \mathrm{yr}$ from 1955.5 to 1969.0. The data of (UT2-A3) are due to the BIH, and those of $x$ and $y$ are due to Vicente and Yumi (1969).

As for the treatment of (UT2-A3) data, the longer period terms are eliminated, before applying the spectrum calculation, by fitting the following formula,

$$
z=a+b t+c t^{2}+e_{1} \sin \Omega+f_{1} \cos \Omega+e_{2} \sin 2 \Omega+f_{2} \cos 2 \Omega
$$

where $\Omega$ is written for the longitude of lunar ascending node. After this, UT2 is converted to UT1 by removing the values of $\Delta T S$ already applied. The annual term which has the largest amplitude is first determined from the spectrum of these modified data, and the semi-annual term is determined next from the spectrum of the residual data after subtracting the annual component, and so on. The annual, semi-annual, and biennial ( 26 month) period terms thus obtained in succession are given below, when 
$t$ is reckoned from 1960.0 in years,

\begin{tabular}{|c|c|}
\hline $\begin{array}{l}19.9^{\mathrm{ms}} 85 \sin 2 \pi t / T \\
\pm 0.74\end{array}$ & $+8 \cdot^{\mathrm{ms}} 27 \cos 2 \pi t / T$ \\
\hline $\begin{aligned} & 6.70 \sin 4 \pi t / T \\
+ & 0.63\end{aligned}$ & $-5.32 \cos 4 \pi t / T$ \\
\hline $\begin{array}{l}-9.27 \sin 2 \pi t /(2.14 T) \\
\pm 0.47\end{array}$ & $-1.71 \cos 2 \pi t /(2.14 T)$ \\
\hline
\end{tabular}

The 2.14 yr period term in the last line confirms the existence of a 26 month period term which was reported first by Iijima and Okazaki (1966).

For the treatment of the $x, y$ data, the spectrum calculations are carried out iteratively in order to separate exactly the Chandler and annual terms which are rather close in period and rather similar in amplitude. The Chandler and annual terms thus obtained are given as below for $x$ and $y$ in order,

$$
\begin{aligned}
& x\left\{\begin{array}{l}
-0.076 \sin 2 \pi t /(1.193 T)+0.147 \cos 2 \pi t /(1.193 T) \\
\pm 0.005 \\
-0.079 \sin 2 \pi t /(1.004 T)-0.068 \cos 2 \pi t /(1.004 T) \\
\pm 0.005
\end{array}\right. \\
& y\left\{\begin{array}{l}
-0.154 \sin 2 \pi t /(1.190 T)-0.072 \cos 2 \pi t /(1.190 T) \\
\pm 0.005 \\
0.059 \sin 2 \pi t /(1.006 T)-0.051 \cos 2 \pi t /(1.006 T) \\
\pm 0.005
\end{array}\right.
\end{aligned}
$$

Minor amplitude terms other than the principal ones mentioned above are found with periods of $0.48,0.86,1.36,1.58,3$, and $4 \mathrm{yr}$ in the UT1, and $1.12,1.26$, and $1.43 \mathrm{yr}$ in the polar motion. It is proved that no periodic term with period common to the UT1 and the polar motion exists except the annual term.

An estimation of the effect of polar motion on the rate of rotation is also attempted. It $l, m$, and $n$ denote the direction cosines of the instantaneous pole as seen from the center of gravity relative to rectangular axes taken parallel to the principal axes of inertia, (those for moments $A, B$, and $C$ respectively) then the fractional rate of change, $\Delta \omega / \omega$, can be expressed as follows,

$$
\Delta \omega / \omega=\left(l^{2}+m^{2}\right)(C-A) / C-m^{2}(C-A) / C \cdot(B-A) /(C-A) .
$$

Apparently, the first term on the right has a period of $6 \mathrm{yr}$ and the second term 0.5 and $0.6 \mathrm{yr}$, because $l$ and $m$ are near to $x$ and $-y$ respectively. If it is assumed here that the amplitude of $\sqrt{l^{2}+m^{2}}$ and $|m|$ are less than $1^{\prime \prime}$ and $(C-A) / C$ and $(B-A) /(C-A)$ are of the order of $3 \times 10^{-3}$ and $1 \times 10^{-1}$ respectively, then the amplitude of the first term becomes less than $0.8 \times 10^{-13}$ and that of the second term $0.1 \times 10^{-13}$. These values in $\Delta \omega / \omega$ correspond to 2.5 and $0.02 \mu \mathrm{sec}$ in time for the first and second terms 
respectively, which are negligibly small. It is proved therefore that no term of the Chandler period can appear in UT1 from the observational as well as from the theoretical point of view.

\section{References}

Iijima, S. and Okazaki, S.: 1966, J. Geod. Soc. Japan 12, No. 2.

Vicente, R. O. and Yumi, S.: 1969, Publ. Int. Lat. Obs. Mizusawa VII, No. 1. 